\title{
Knowing Too Much
}

\section{Geoff Goodfellow}

Even by the time some reach

five years of age

they know all too much

about stormy weather

you'll often see it in the way

they play their games

in their Charlie Chaplin moves

once they have pressed

the mute button

by the sound of their footsteps -

by the sounds of others footsteps

in where they go for hide \& seek

$\&$ how fast they run for chasey

by the curl of their lip -

by the fall of their tears. 